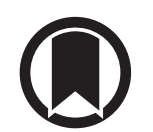

CrossMark

\title{
Why does oxygen supplementation during exercise training in COPD patients with exercise-induced desaturation not consistently improve exercise capacity?
}

\begin{abstract}
To the Editor:
We would like to congratulate the authors of the recent study entitled "Oxygen compared to air during exercise training in COPD with exercise-induced desaturation" for conducting a methodologically well-designed randomised controlled trial (RCT) [1]. The potential effects of several adjuncts to exercise training during pulmonary rehabilitation, such as supplemental oxygen or inspiratory muscle training, are still a matter for debate [2]. Additional improvements in functional capacity are only likely to occur when the adjunct interventions enable patients to train at higher intensities during their general exercise-training programme. There exists a sound physiological rationale for correcting hypoxaemia as a strategy to improve performance and reduce symptoms during exercise in patients with COPD [3]. However, not all of the previous randomised clinical trials were able to achieve relevant contrasts in training intensities and treatment outcomes between oxygen and air groups [4].
\end{abstract}

The study by Auson et al. [1] constitutes the largest RCT conducted in this area so far. It confirms that supplemental oxygen was not required for patients to train safely and to derive some benefits from exercise training. When looking closer at the results it appears, however, that the room air group did not succeed in achieving clinically relevant improvements in the shuttle walk test after an intensive 8-week training period. It is surprising that the oxygen group was not able to exceed the improvements achieved by the room air group. The authors explain the absence of between group differences in the primary outcome by concluding that "during treadmill training the oxygen group was not able to achieve a greater training dose per session than the [room] air group, despite a significantly higher measured $S_{\mathrm{pO}_{2}}$ and significantly lower dyspnoea and RPE [rating of perceived exertion] scores during treadmill training sessions." This inability to train at higher intensities (despite higher $S_{\mathrm{pO}_{2}}$ and lower symptom scores) is unexpected given the description of the planned progression of exercise intensity during the training programme. The authors initially aimed to "increase training intensity according to symptoms so that dyspnoea or rate of perceived exertion would be maintained at a "moderate" to "somewhat severe" level (i.e. a score of 3 to 4 on the modified Borg dyspnoea and RPE (0-10) scales)" [5]. First, these target symptom scores appear to be rather low in comparison with previous recommendations $[6,7]$. In addition, when looking at actual average Borg scores that were recorded during treadmill walking sessions (table $S 1$ in their supplementary material) it appears that a large part of the oxygen group must have been training at scores well below these pre-specified targets. Reported standard deviations around average Borg scores indicate that about half of the oxygen group must have trained at scores between $1(!)$ and 3 . In contrast, during the initial endurance shuttle walk test subjects were able to walk for longer than $5 \mathrm{~min}$ on room air with Borg scores above 4 on average. This of course raises the question as to why these patients were not able to tolerate higher symptom scores during their walking training sessions on the treadmill? Was the fact that training intensity could only be increased every three sessions (once a week) possibly preventing subjects from reaching sufficient increases in training intensity? Or was it the fact that both dyspnoea and RPE scores had to be $<3$ after a treadmill training session before intensity could be increased? Or were the physiotherapists (who were blinded to group allocation) maybe just overly cautious to increase intensity during the training sessions?

@ERSpublications

Additional improvements in functional capacity are only likely to occur when adjunct interventions applied during pulmonary rehabilitation enable patients to train at higher intensities during their general exercise-training programme http://bit.ly/2TMxEel

Cite this article as: Langer D, Gosselink R. Why does oxygen supplementation during exercise training in COPD patients with exercise-induced desaturation not consistently improve exercise capacity? Eur Respir J 2019; 54: 1901586 [https://doi.org/10.1183/13993003.01586-2019]. 
Another interesting observation from the current study is that some differences in training intensity could be achieved between the oxygen and the room air group during exercise on the bicycle in contrast to treadmill walking. Since patients had to perform their exercises as continuous training (without interruptions) we were wondering whether this might have been related to the differences in initial training durations (cycling sessions 10 min versus treadmill $20 \mathrm{~min}$ )? Would it in hindsight maybe have been better to subdivide the treadmill training into blocks of 5 to $10 \mathrm{~min}$ ? Or might cycling training simply be the preferred training modality in this context? We believe that some additional discussion on the points raised above could provide important information for future work in this area.

Daniel Langer $\oplus^{1,2}$ and Rik Gosselink $\oplus^{1,2}$

${ }^{1}$ Respiratory Rehabilitation and Respiratory Division, University Hospital Leuven, Leuven, Belgium. ${ }^{2}$ Dept of Rehabilitation Sciences, KU Leuven, Leuven, Belgium.

Correspondence: Daniel Langer, Dept of Rehabilitation Sciences, KU Leuven, Herestraat 49, 3000 Leuven, Belgium. E-mail: daniel.langer@kuleuven.be

Received: 9 Aug 2019 | Accepted: 13 Aug 2019

Conflict of interest: D. Langer has nothing to disclose. R. Gosselink has nothing to disclose.

\section{References}

1 Alison JA, McKeough ZJ, Leung RWM, et al. Oxygen compared to air during exercise training in COPD with exercise-induced desaturation. Eur Respir J 2019; 53: 1802429.

2 Camillo CA, Osadnik CR, van Remoortel H, et al. Effect of "add-on" interventions on exercise training in individuals with COPD: a systematic review. ERJ Open Res 2016; 2: 00078-2015.

3 Dilektasli AG, Porszasz J, Stringer WW, et al. Physiologic effects of oxygen supplementation during exercise in chronic obstructive pulmonary disease. Clin Chest Med 2019; 40: 385-395.

4 Nonoyama ML, Brooks D, Lacasse Y, et al. Oxygen therapy during exercise training in chronic obstructive pulmonary disease. Cochrane Database Syst Rev 2007; 2: CD005372.

5 Alison JA, McKeough ZJ, Jenkins SC, et al. A randomised controlled trial of supplemental oxygen versus medical air during exercise training in people with chronic obstructive pulmonary disease: supplemental oxygen in pulmonary rehabilitation trial (SuppORT) (Protocol). BMC Pulm Med 2016; 16: 25.

6 Horowitz MB, Littenberg B, Mahler DA. Dyspnea ratings for prescribing exercise intensity in patients with COPD. Chest 1996; 109: 1169-1175.

7 Spruit MA, Singh SJ, Garvey C, et al. An official American Thoracic Society/European Respiratory Society statement: key concepts and advances in pulmonary rehabilitation. Am J Respir Crit Care Med 2013; 188: e13-e64.

\section{Exercise training in COPD with exercise- induced desaturation does improve exercise capacity, irrespective of whether supplemental oxygen or air is provided during training}

From the authors:

We thank D. Langer and R. Gosselink for their correspondence about our study [1]. They raise some interesting points, mainly related to training intensity.

We contend that the room air group did achieve a clinically relevant improvement in the incremental shuttle walk test, since the minimal important difference of $36.1 \mathrm{~m}$ [2] fell within the $95 \%$ confidence interval for mean improvement for both air and oxygen groups, being 13 to $42 \mathrm{~m}$ and 20 to $47 \mathrm{~m}$, respectively. 
The training intensity was a target symptom score of 3 to 4 which is within the range recommended in clinical practice [3] and was chosen for the current study based on work from our group showing that for people with COPD, exercising on a cycle ergometer at intensities corresponding to 3 to 4 ("moderate" to "somewhat severe") for dyspnoea or rate of perceived exertion (RPE) (whichever was the higher) was equivalent to $78 \%$ peak oxygen uptake $\left(V_{\mathrm{O}_{2}}\right)$ or $71 \% V_{\mathrm{O}_{2}}$ reserve [4]. Training at intensities of $60-85 \% V_{\mathrm{O}_{2}}$ peak or $40-85 \% V_{\mathrm{O}_{2}}$ reserve has been recommended in major reviews [5] and our previous work has shown that progressing walking training based on eliciting a dyspnoea score of at least 3 achieved significant improvements in exercise capacity [6]. While it is true that participants achieved higher symptom scores in the endurance shuttle walk test (ESWT) than during training, this was to be expected as the ESWT was symptom limited while training was at sub-maximal levels.

Data in our table S1 represented the average symptom scores. Review of participants' scores for each training session (1947 sessions available for review out of a possible 2328) revealed that only five participants in the air group and 10 in the oxygen group had both dyspnoea and RPE scores $<3$ for six or more sessions (i.e. for more than 2 weeks of training), with most of these "lower intensity" training sessions occurring in the early part of the training programme. These participants may have been able to train at a slightly higher intensity but as they represent a relatively small number we are confident that, overall, the training intensity was sufficient and that this is unlikely to be the reason that the oxygen group did not have a greater training response than the air group.

While there were statistically significantly lower symptom scores in the oxygen than in the air group during training, these differences were small $(<1$ point) and not clinically meaningful, since 2 points is the estimated clinical meaningful difference [7]. Such small differences were unlikely to be of sufficient magnitude to be perceived by participants or to translate into differences in the training intensities.

D. Langer and R. Gosselink queried whether the physiotherapists may have been overly cautious in increasing the intensity, as they were blind to group allocation. We believe that this blinding was a strength of our study since the clinicians increased the training intensity based on the participants' symptoms and were not influenced by their beliefs in the benefits or safety of oxygen supplementation.

We did not use intermittent blocks of training in the treadmill or cycle training so it is unknown whether this would have resulted in a higher training intensity for both groups or would have advantaged the oxygen group.

Our study showed that a significantly greater training volume was achieved during cycle training by the oxygen group compared to the air group, which was not demonstrated in the walking training. One possible explanation is that cycling uses a smaller muscle mass than walking and produces higher lactic acid for a similar $V_{\mathrm{O}_{2}}$, the buffering of which increases carbon dioxide levels, resulting in a greater ventilatory stimulus. Using supplemental oxygen to reduce ventilation at submaximal work rates could delay hyperinflation, reduce dyspnoea [8] and facilitate tolerance of a larger training dose. If this occurred in any of our participants it did not translate into better training outcomes as measured by walk tests. Had we used a cycle test as an outcome measure, it is possible there may have been a difference between the oxygen and air groups. Previous small studies comparing oxygen and air supplementation during exercise training in people with COPD who desaturated during walking exercise showed no significant differences between groups, with walking tests used as an outcome measure [9]. We do not believe that cycle training is the preferred training mode as cycle training alone may not address the goal of improving capacity for functional daily tasks to the same extent as walking training [10].

Our large randomised controlled trial, and previous smaller studies, demonstrate that the physiological benefits of training with oxygen are small and largely overwhelmed by the training response, which in our study resulted in improved exercise capacity and health-related quality of life for both groups, with no differences between groups.

@ERSpublications

In people with COPD who desaturate during exercise, similar improvements in exercise capacity and health-related quality of life can be achieved with exercise training, irrespective of whether supplemental oxygen or air is provided during training http://bit.ly/2kKGGvi

Cite this article as: Alison JA, McKeough ZJ, Leung RWM, et al. Exercise training in COPD with exercise-induced desaturation does improve exercise capacity, irrespective of whether supplemental oxygen or air is provided during training Eur Respir J 2019; 54: 1901725 [https://doi.org/10.1183/13993003.017252019]. 
Jennifer A. Alison $\oplus^{1,2}$, Zoe J. McKeough ${ }^{1}$, Regina W.M. Leung ${ }^{3}$, Anne E. Holland $\oplus^{4,5,6}$, Kylie Hill, Norman R. Morris ${ }^{9,10}$, Sue Jenkins $s^{7,8,11}$, Lissa M. Spencer ${ }^{12}$, Catherine J. Hill ${ }^{6,13}$, Annemarie L. Lee ${ }^{14}$, Helen Seale ${ }^{15}$, Nola Cecins ${ }^{11}$ and Christine F. McDonald ${ }^{6,16}$

${ }^{1}$ Faculty of Health Sciences, Discipline of Physiotherapy, The University of Sydney, Sydney, Australia. ${ }^{2}$ Sydney Local Health District, Sydney, Australia. ${ }^{3}$ Concord Repatriation General Hospital, Sydney, Australia. ${ }^{4}$ Discipline of Physiotherapy, School of Allied Health, LaTrobe University, Melbourne, Australia. ${ }^{5}$ Dept of Physiotherapy, Alfred Health, Melbourne, Australia. ${ }^{6}$ Institute for Breathing and Sleep, Melbourne, Australia. ${ }^{7}$ School of Physiotherapy and Exercise Science, Faculty of Health Sciences, Curtin University, Perth, Australia. ${ }^{8}$ Institute for Respiratory Health, Sir Charles Gairdner Hospital, Perth, Australia. ${ }^{9}$ School of Allied Health Sciences, Griffith University, Southport, Australia.

${ }^{10}$ Metro North Hospital and Health Service, The Prince Charles Hospital, Allied Health Research Collaborative, Brisbane, Australia. ${ }^{11}$ Dept of Physiotherapy, Sir Charles Gardiner Hospital, Perth, Australia. ${ }^{12}$ Dept of Physiotherapy, Royal Prince Alfred Hospital, Sydney, Australia. ${ }^{13}$ Dept of Physiotherapy, Austin Health, Melbourne, Australia. ${ }^{14}$ School of Allied Health, La Trobe University, Melbourne, Australia. ${ }^{15}$ Dept of Physiotherapy, Prince Charles Hospital, Queensland, Australia. ${ }^{16}$ Dept of Respiratory and Sleep Medicine, Austin Health, Melbourne, Australia.

Correspondence: Jennifer A. Alison, Discipline of Physiotherapy, Faculty of Health Sciences, The University of Sydney, 75 East St, Lidcombe NSW 2141, Australia. E-mail: jennifer.alison@sydney.edu.au

Received: 1 Sept 2019 | Accepted after revision: 13 Sept 2019

Conflict of interest: J.A. Alison reports grants from National Health and Medical Research Council, Australia, during the conduct of the study. Z.J. McKeough reports grants from National Health and Medical Research Council, Australia, during the conduct of the study. R.W.M. Leung reports grants and personal fees from National Health and Medical Research Council, Australia, during the conduct of the study. A.E. Holland reports grants from National Health and Medical Research Council, Australia, during the conduct of the study. K. Hill grants from National Health and Medical Research Council, Australia, during the conduct of the study; personal fees for lectures from Menarini Australia, personal fees for meeting attendance from Journal of Physiotherapy, grants from Better Breathing Foundation, royalties from SLACK publishing, outside the submitted work. N.R. Morris reports grants from National Health and Medical Research Council, Australia, during the conduct of the study. S. Jenkins reports grants from National Health and Medical Research Council, Australia, during the conduct of the study. L.M. Spencer reports grants from National Health and Medical Research Council, Australia, during the conduct of the study. C.J. Hill reports grants from National Health and Medical Research Council, Australia, during the conduct of the study. A.L. Lee reports grants from National Health and Medical Research Council, Australia, during the conduct of the study. H. Seale reports grants from National Health and Medical Research Council, Australia, during the conduct of the study. N. Cecins reports grants from National Health and Medical Research Council, Australia, during the conduct of the study. C.F. McDonald reports personal fees from Pfizer, GSK and Novartis, payment for lectures to hospital from Menarini, outside the submitted work.

\section{References}

1 Alison JA, McKeough ZJ, Leung RWM, et al. Oxygen compared to air during exercise training in COPD with exercise-induced desaturation. Eur Respir J 2019; 53: 1802429.

2 Evans RA, Singh SJ. Minimum important difference of the incremental shuttle walk test distance in patients with COPD. Thorax 2019; 74: 994-995.

3 Jenkins S, Hill K, Cecins NM. State of the art: how to set up a pulmonary rehabilitation program. Respirology 2010; 15: 1157-1173.

4 Zainuldin R, Mackey MG, Alison JA. Prescribing cycle exercise intensity using moderate symptom levels in chronic obstructive pulmonary disease. J Cardiopulm Rehabil Prev 2016; 36: 195-202.

5 Troosters T, Casaburi R, Gosselink R, et al. State of the Art. Pulmonary rehabilitation in chronic obstructive pulmonary disease. Am J Respir Crit Care Med 2005; 172: 19-38.

6 Wootton SL, Ng LW, McKeough ZJ, et al. Ground-based walking training improves quality of life and exercise capacity in COPD. Eur Respir J 2014; 44: 885-894.

7 Crisafulli E, Clini EM. Measures of dyspnea in pulmonary rehabilitation. Multidiscip Respir Med 2010; 5: 202-210.

8 O'Donnell DE, D'Arsigny C, Webb KA. Effects of hyperoxia on ventilatory limitation during exercise in advanced chronic obstructive pulmonary disease. Am J Respir Crit Care Med 2001; 163: 892-898.

9 Nonoyama ML, Brooks D, Lacasse Y, et al. Oxygen therapy during exercise training in chronic obstructive pulmonary disease. Cochrane Database Syst Rev 2007; 2: CD005372.

10 Leung RWM, Alison JA, McKeough ZJ, et al. Ground walk training improves functional exercise capacity more than cycle training in people with chronic obstructive pulmonary disease (COPD): a randomised trial. J Physiother 2010; 56: 105-112 